Electronic Supporting information of the article

\title{
Molecular spin crossover in slow motion: Light-induced spin- state transitions in trigonal prismatic iron(II) complexes
}

Phillip Stock, Eva Deck, Silvia Hohnstein, Jana Korzekwa, Karsten Meyer, Frank W. Heinemann, Frank Breher and Gerald Hörner

Technische Universität Berlin, Institut für Chemie, Straße des 17. Juni 135, 10623 Berlin, Germany; Institute of Inorganic Chemistry, Karlsruhe Institute of Technology (KIT),

Engesserstrasse 15, 76131 Karlsruhe, Germany; Department Chemie und Pharmazie, Universität Erlangen-Nürnberg, Egerlandstraße 1, 91058 Erlangen, Germany.

SQUID data and Mössbauer spectrum of $1(\mathrm{OTf})_{2}$

Reference polyhedra

Structure details

Continuous shape map

Thermodynamics of SCO of $2\left(\mathrm{BF}_{4}\right)_{2}$

Transient decay profiles of $2\left(\mathrm{BF}_{4}\right)_{2}$ 7

Transient absorption spectra of $2\left(\mathrm{BF}_{4}\right)_{2}$ 8

Activation parameters of $\mathrm{SCO}$ in $2\left(\mathrm{BF}_{4}\right)_{2}$ 9

Cartesian coordinates of the DFT structures of $[1]^{2+}-[4]^{2+}$ 10 
(a)

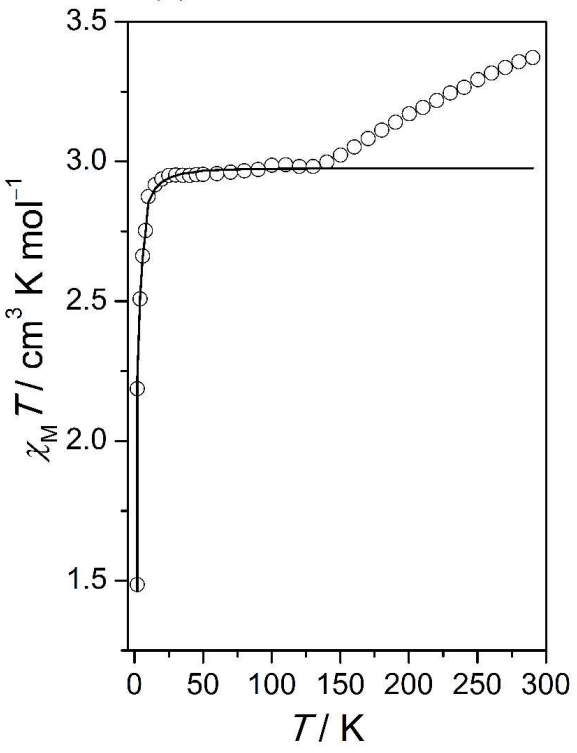

(b)

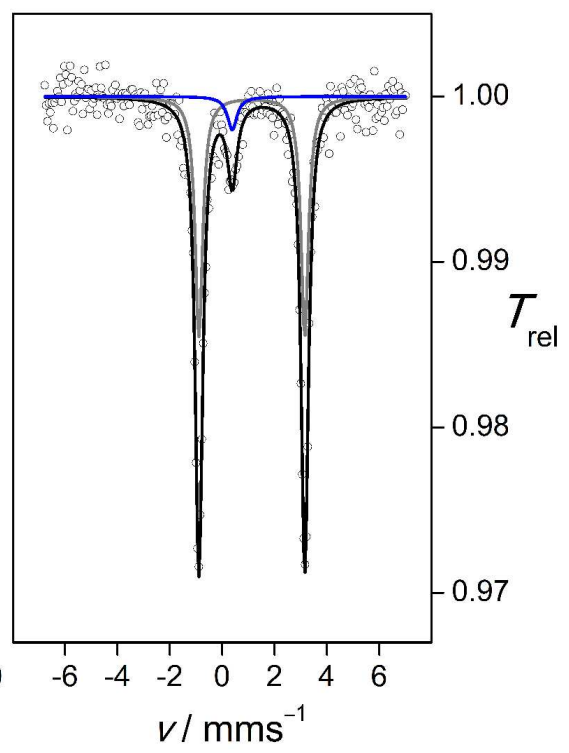

Figure S1. (a) Solid-state $\chi_{\mathrm{M}} T / T$ diagram (2-300 K) for $1(\mathrm{OTf})_{2}$ (line: fit for data points at $T<$ $120 \mathrm{~K}$ with $\mathrm{g}=2.00,|D|=4.749 \mathrm{~cm}^{-1}, E / D=0.0$; diamagnetic correction $=-965.3 \times 10^{-6} \mathrm{emu}$ );

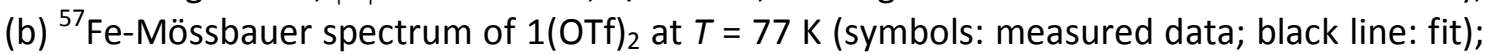
grey (doublet): $\delta=1.13(1) \mathrm{mm} \mathrm{s}^{-1} ; \Delta E_{\mathrm{Q}}=4.04(1) \mathrm{mm} \mathrm{s}^{-1} ; \Gamma_{\mathrm{FWHM}}=0.35(1) \mathrm{mm} \mathrm{s}^{-1}$; blue (singlet): $\delta=0.39(1) \mathrm{mm} \mathrm{s}^{-1} ; \Gamma_{\mathrm{FWHM}}=0.44(1) \mathrm{mm} \mathrm{s}^{-1}$.

Discussion of Figure S1: The samples of $\mathbf{1}(\mathrm{OTf})_{2}$ gave evidence of minor contamination. In its Mössbauer spectrum a second component contributes with ca. $10 \%$ weight. Its small isomeric shift $\left(\delta=0.39(1) \mathrm{mm} \mathrm{s}^{-1}\right)$ and the lack of quadrupole splitting clearly identify this component as being due to Is-iron(II) in a fairly undisturbed octahedral environment. Although the chemical nature of this component cannot be unequivocally assigned at present, it is tempting to attribute this signal to the $I s$-isomer of $\mathbf{1}(\mathrm{OTf})_{2}$. Firstly, the analytical data, i.e., the data from elemental analysis, are incompatible with the presence of a severe contamination by iron(II) complexes with deviating constitution. Secondly, the Mössbauer parameters of the Is-iron(II) component are highly reminiscent of octahedral Is-iron(II) complexes that derive from pyrimidine analogues of ligand $\mathbf{L}^{2}{ }^{[1]}$ However, SQUID measurements between $2-300 \mathrm{~K}$ clearly show no SCO for $\mathbf{1}(\mathrm{OTf})_{2}$ in the solid state over the whole temperature range.

\footnotetext{
${ }^{1}$ E. Deck, J. Korzekwa, K. Meyer, G. Hörner, F. Breher, unpublished results.
} 

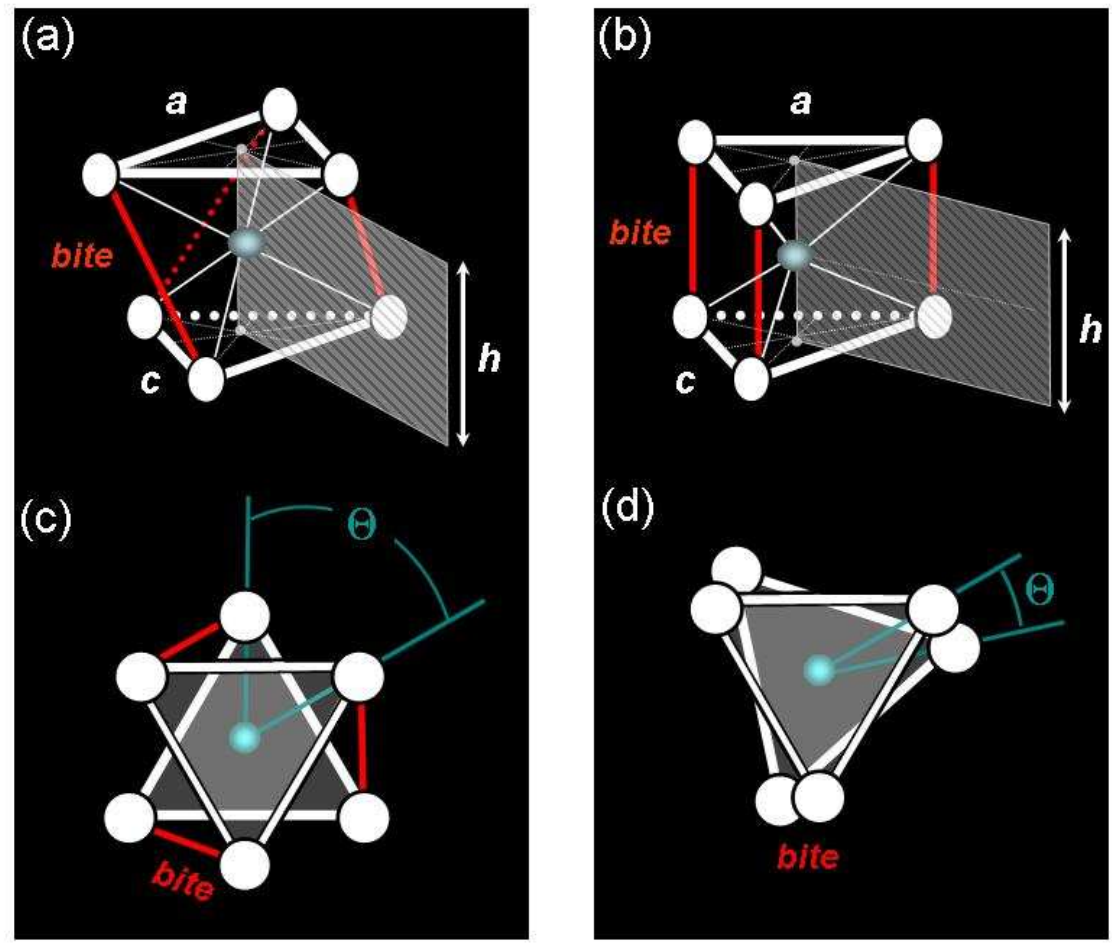

Figure S2. Selected geometric measures for complexes of tris-bidentate ligands; $(a, c)$ for the case of the octahedron; (b, d) for the trigonal prismatic structure; $h$ : height of the polyhedron (here distance of the planes defined by $\left(\mathrm{N}_{\mathrm{imin}}\right)_{3}$ and $\left.\left(\mathrm{N}_{\text {het }}\right)_{3}\right)$; $a$ : non-bonded distance of $\mathrm{N}_{\text {imin }}$ donors; $c$ : non-bonded distance of $\mathrm{N}_{\text {het }}$ donors; bite: intra-podal donor distance; $\theta$ : trigonal twist angle of the planes $\left(\mathrm{N}_{\mathrm{imin}}\right)_{3}$ and $\left(\mathrm{N}_{\text {het }}\right)_{3}$. 
Table S1. Selected structural details (bond lengths, nonbonded distances $(\mathrm{pm})$ and angles $\left({ }^{\circ}\right)$ ) for $\mathbf{1}(\mathrm{X})_{2}$ and $\mathbf{2}\left(\mathrm{BF}_{4}\right)_{2}$, and for DFT-derived structures of [ $\left.\mathrm{FeL}^{n}\right]$.

\begin{tabular}{|c|c|c|c|c|}
\hline XRD & $\begin{array}{l}\mathbf{1}\left(\mathrm{BF}_{4}\right)_{2}{ }^{a} \\
\mathrm{Fe} 1\end{array}$ & $\mathrm{Fe} 5$ & $1(\mathrm{OTf})_{2}$ & $2\left(\mathrm{BF}_{4}\right)_{2}$ \\
\hline \multicolumn{5}{|c|}{ (Mean) Distances } \\
\hline $\bar{d}\left(\mathrm{Fe}-\mathrm{N}_{\mathrm{imin}}\right)$ & $223.9(2)$ & $224.5(5)$ & $225.5(20)$ & $226.2(1)$ \\
\hline $\bar{d}$ Fe- $\left.\mathrm{N}_{\text {het }}\right)$ & $213.8(4)$ & $214.2(8)$ & $213.1(6)$ & 212.0(1) \\
\hline$h^{b}$ & 262.0 & 265.4 & 265.2 & 264.0 \\
\hline$h_{1}^{b}$ & 145.6 & 146.6 & 148.9 & 149.3 \\
\hline$h_{2}^{b}$ & 116.4 & 118.8 & 116.3 & 114.7 \\
\hline bite ${ }^{b}$ & $265.7(17)$ & $266.3(5)$ & $265.7(11)$ & $264.8(1)$ \\
\hline$a^{b}$ & $294.6(27)$ & 294.2(29) & $293.2(40)$ & $294.4(4)$ \\
\hline$c^{b}$ & $310.9(11)$ & $308.6(9)$ & $309.2(32)$ & $308.9(1)$ \\
\hline \multicolumn{5}{|l|}{ Angles } \\
\hline$(\mathrm{N}-\mathrm{Fe}-\mathrm{N})_{\text {trans }}$ & $143.2(11)$ & $137.0(11)$ & $135.6(20)$ & $137.2(5)$ \\
\hline$\Sigma_{c i s}^{c}$ & 180.2 & 191.7 & 190.4 & 188.6 \\
\hline$\theta^{b}$ & $14.7(5)$ & $6.0(12)$ & $4.2(8)$ & $6.6(1)$ \\
\hline $\mathrm{DFT}^{d}$ & {$[1]^{2+}$} & {$[2]^{2+}$} & {$[3]^{2+}$} & {$[4]^{2+}$} \\
\hline \multicolumn{5}{|c|}{ (Mean) Distances } \\
\hline \multirow[t]{2}{*}{$d\left(\mathrm{Fe}-\mathrm{N}_{\mathrm{imin}}\right)$} & $224.8(18)$ & $230.3(20)$ & $221.7(10)$ & $218.5(7)$ \\
\hline & 198.0(4) & 198.2(4) & $196.0(1)$ & $200.5(1)$ \\
\hline \multirow[t]{2}{*}{$d\left(\mathrm{Fe}-\mathrm{N}_{\text {het }}\right)$} & $216.8(14)$ & $212.8(11)$ & $218.8(12)$ & $225.9(12)$ \\
\hline & 199.0(2) & $197.5(3)$ & $200.8(2)$ & 199.0(1) \\
\hline \multirow[t]{2}{*}{$h^{c}$} & 257.4 & 262.6 & 259.0 & 223.7 \\
\hline & 225.7 & 227.9 & 226.5 & 209.5 \\
\hline \multirow[t]{2}{*}{$h_{1}{ }^{b}$} & 145.3 & 153.2 & 142.1 & 88.0 \\
\hline & 114.6 & 114.6 & 114.2 & 102.5 \\
\hline \multirow[t]{2}{*}{$h_{2}^{b}$} & 112.1 & 109.4 & 116.9 & 135.7 \\
\hline & 111.1 & 113.3 & 112.3 & 107.0 \\
\hline \multirow[t]{2}{*}{ bite $^{b}$} & $268.3(10)$ & $268.9(20)$ & $267.5(12)$ & $270.2(11)$ \\
\hline & $256.8(5)$ & $255.8(1)$ & $257.0(3)$ & 259.1(1) \\
\hline \multicolumn{5}{|l|}{ Angles } \\
\hline \multirow[t]{2}{*}{$(\mathrm{N}-\mathrm{Fe}-\mathrm{N})_{\text {trans }}$} & $149.6(18)$ & $145.4(22)$ & $148.0(18)$ & $162.4(7)$ \\
\hline & $166.8(5)$ & $165.4(10)$ & $166.6(3)$ & $173.9(1)$ \\
\hline \multirow[t]{2}{*}{$\Sigma_{c i s}^{c}$} & 155.4 & 175.8 & 156.6 & 103.7 \\
\hline & 62.0 & 58.3 & 63.3 & 60.6 \\
\hline \multirow[t]{2}{*}{$\theta^{b}$} & $24.0(15)$ & $18.9(21)$ & $21.2(20)$ & $46.6(6)$ \\
\hline & $44.1(2)$ & $42.1(8)$ & $43.7(4)$ & $53.1(1)$ \\
\hline
\end{tabular}

${ }^{a}$ Representative data for complex ions with Fe 1 and Fe $5,{ }^{b}$ see Figure S2 for definitions; $h_{1}$ and $h_{2}$ denote the 
distance of the central metalion from the planes defined by $\left(\mathrm{N}_{\mathrm{imin}}\right)_{3}$ and $\left(\mathrm{N}_{\text {het }}\right)_{3}$, respectively; ${ }^{c}$ summed deviation from $90^{\circ}$ of twelve N-Fe-N cis angles; ${ }^{d}$ fully optimized structures of $h s-\left[\right.$ FeL $\left.^{n}\right]$ (B3LYP*/TZVP); data for the $l s$-states in italics.

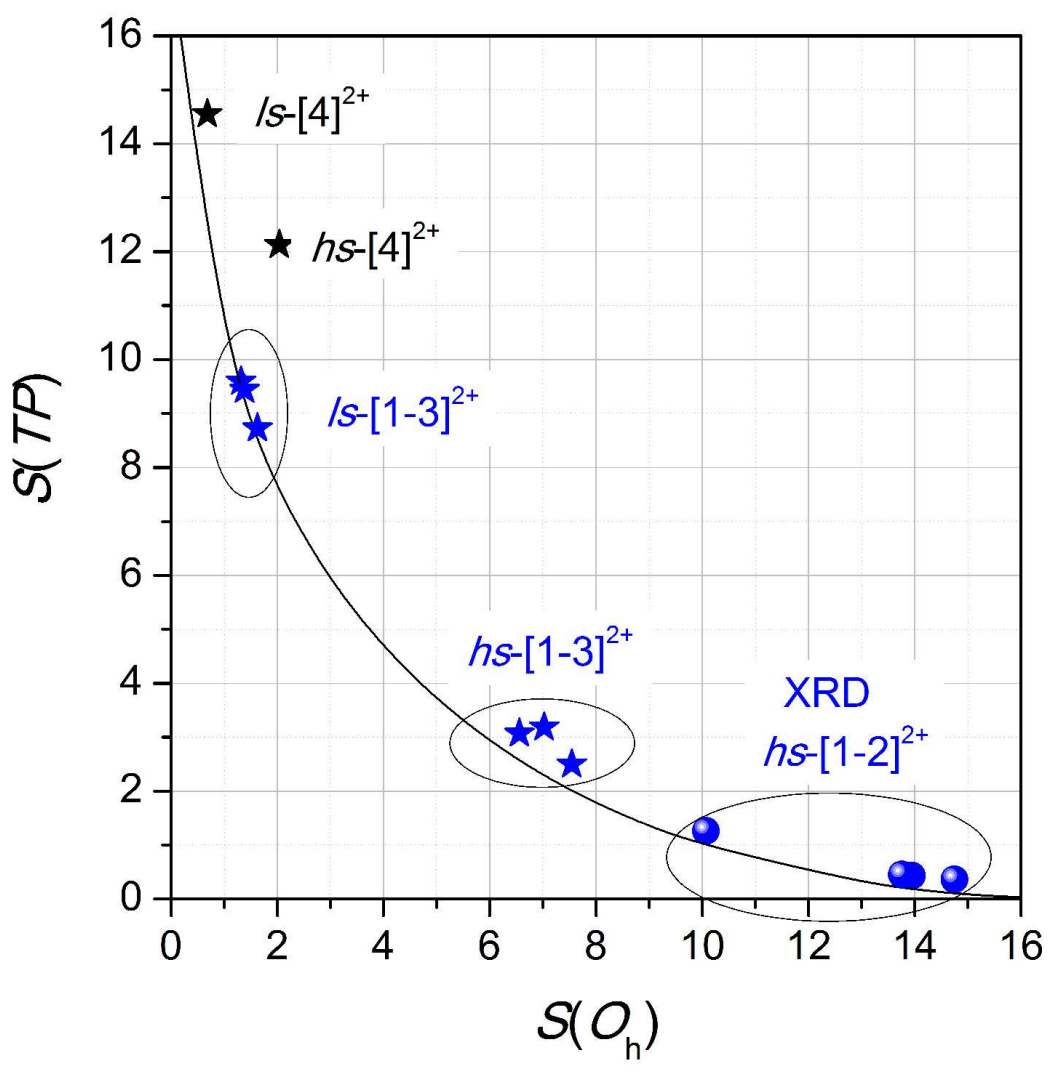

Figure S3. Continuous shape map of the octahedron $\leftrightarrow$ trigonal prism transition; data from Table 1; blue: $[\mathbf{1 - 3}]^{2+}$; black: [4] ${ }^{2+}$; line: ideal Bailar twist; balls: XRD-derived structures; stars: DFT-optimised structures (B3LYP*-D/TZVP). 


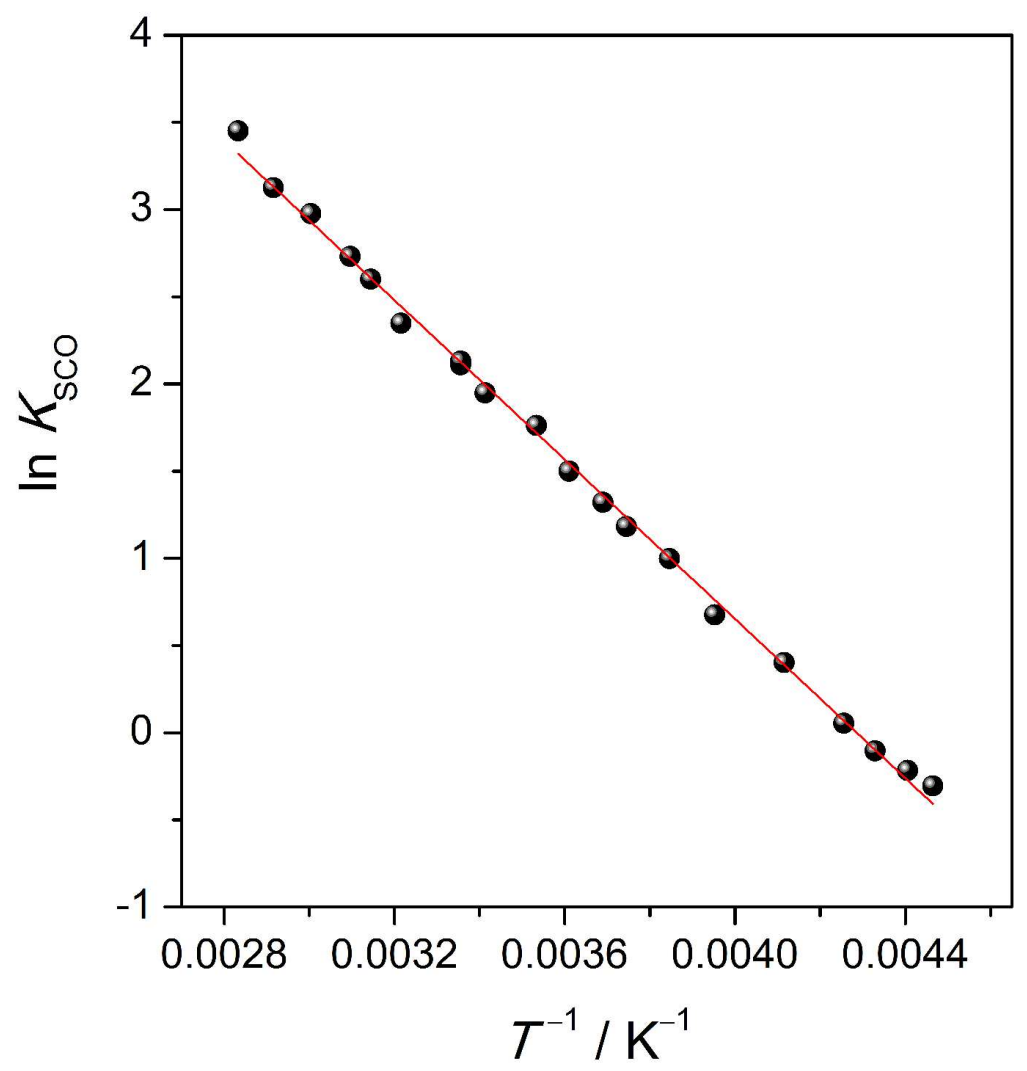

Figure S4. Van't Hoff analysis of the thermochromism of $\mathbf{2}\left(\mathrm{BF}_{4}\right)_{2}$ in 1,1,1-trifluoroethanol; equilibrium constants $K_{\text {sco }}$ derived from the temperature dependent peak absorption at $\lambda_{\text {obs }}$ $=470 \mathrm{~nm}$ assuming molar absorption coefficients $\varepsilon_{\mathrm{HS}}=250 \mathrm{~L} \mathrm{~mol}^{-1} \mathrm{~cm}^{-1}$ and $\varepsilon_{\mathrm{LS}}=8000 \mathrm{~L}$ $\mathrm{mol}^{-1} \mathrm{~cm}^{-1}$ for the $h s$-state and the $I s$-state, respectively. 


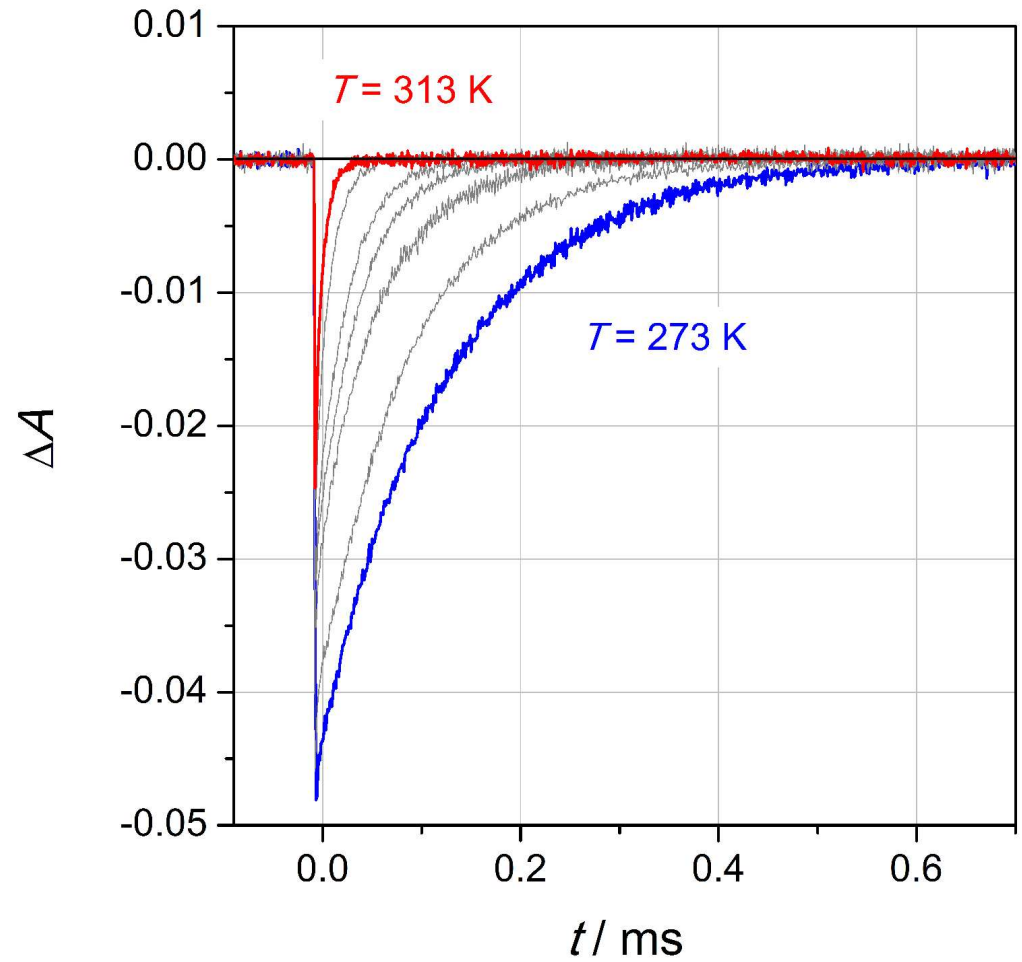

Figure S5. Additional variable-temperature transient recovery profiles of $\mathbf{2}\left(\mathrm{BF}_{4}\right)_{2}$ in $1,1,1-$ trifluoroethanol $\left(\lambda_{\text {obs }}=470 \mathrm{~nm}\right)$. 


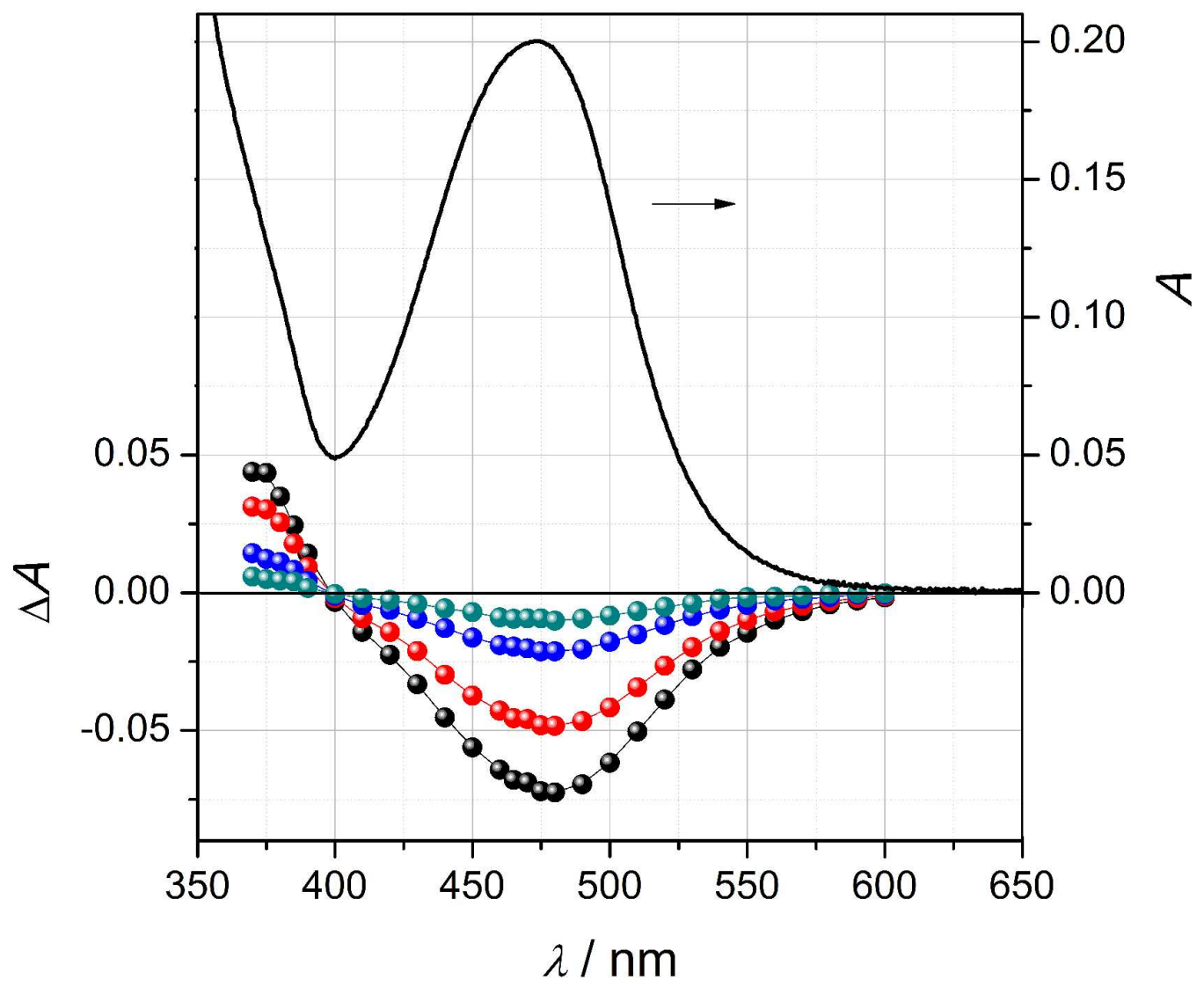

Figure S6. (top) Steady-state UV-Vis spectrum of $2\left(\mathrm{BF}_{4}\right)_{2}$ in 1,1,1-trifluoroethanol at $T=240$ $\mathrm{K}$; (bottom) transient absorption spectra obtained at various times after laser-flash photolysis $\left(\lambda_{\mathrm{exc}}=532 \mathrm{~nm} ; I_{\text {pulse }} \approx 5 \mathrm{~mJ}\right.$ ) of $2\left(\mathrm{BF}_{4}\right)_{2}$ in 1,1,1-trifluoroethanol at room temperature. 


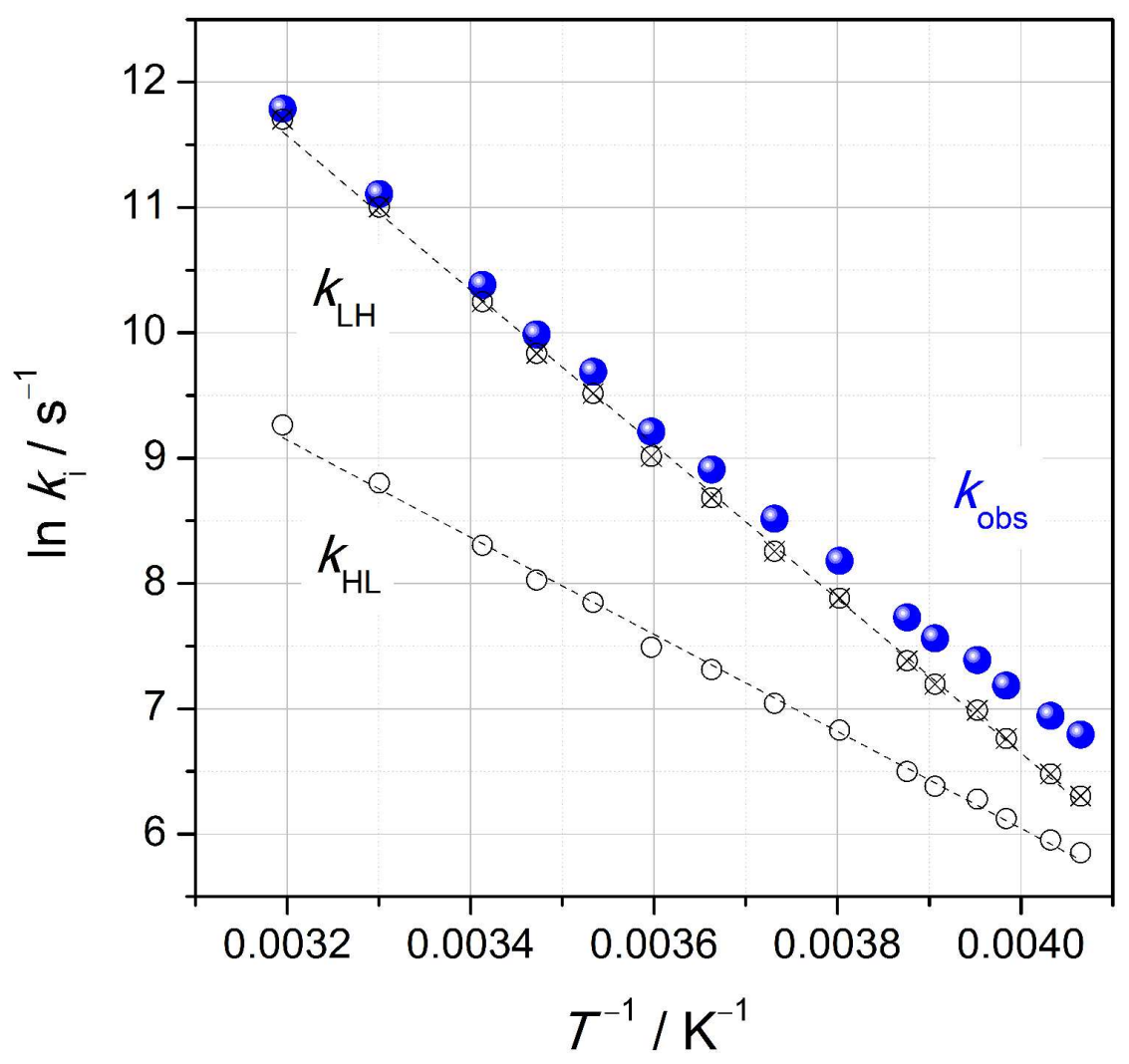

Figure S7. Arrhenius analysis of the spin-state dynamics of $\mathbf{2}\left(\mathrm{BF}_{4}\right)_{2}$ in 1,1,1-trifluoroethanol; the experimental rate constants of re-equilibration of $h s$ and $/ s$ states ( $k_{\text {obs}}$; blue) are split into the components of $h s \rightarrow l s$ ( $k_{\mathrm{HL}}$; open symbols) and $I s \rightarrow h s$ transitions ( $k_{\mathrm{LH}}$; crossed symbols) utilising the thermodynamic parameters derived in Figure S3; fit parameters (i) for $k_{\mathrm{HL}}: A_{0}=(2.3 \pm 0.4) 10^{9} \mathrm{~s}^{-1}, E_{\mathrm{a}}=(32.3 \pm 0.5) \mathrm{kJ} \mathrm{mol}^{-1}$; (ii) for $k_{\mathrm{LH}}: A_{0}=(3.9 \pm 0.9) 10^{13} \mathrm{~s}^{-1}, E_{\mathrm{a}}=$ $(51.2 \pm 0.5) \mathrm{kJ} \mathrm{mol}^{-1}$.

It is noted that the given standard deviations reflect the quality of the fit but not the quality of the underlying data. Although the underlying thermodynamic data are chemically reasonable (see Figure S3), utilization of other sets of thermodynamic parameters, $\Delta_{\mathrm{sco}} H$ and $\Delta_{\mathrm{sco}} \mathrm{S}$, may similarly give rise to decent fits. 
Table S2. Cartesian coordinates of complex $/ s-[\mathbf{1}]^{2+}(S=0$; B3LYP*-D/TZVP).

\begin{tabular}{|c|c|c|c|}
\hline $\mathrm{C}$ & -1.139861 & 0.759320 & -3.902166 \\
\hline C & -1.486744 & -1.097264 & -2.726224 \\
\hline $\mathrm{C}$ & -0.677161 & 0.920374 & -2.618477 \\
\hline C & -0.145131 & 2.024766 & -1.867155 \\
\hline C & 2.185799 & -1.406535 & -1.929049 \\
\hline C & -0.293264 & -3.276992 & 0.187783 \\
\hline $\mathrm{C}$ & 0.568808 & 4.075612 & -0.119760 \\
\hline $\mathrm{C}$ & 3.762604 & -1.570060 & -0.368605 \\
\hline $\mathrm{C}$ & 2.645912 & -0.960990 & 0.150902 \\
\hline C & -2.285211 & -3.312821 & 1.178837 \\
\hline C & -1.886094 & -2.014592 & 0.968866 \\
\hline $\mathrm{C}$ & 2.330621 & -0.333312 & 1.405236 \\
\hline C & -2.392573 & -0.724356 & 1.348880 \\
\hline $\mathrm{C}$ & -3.018969 & 1.845921 & 2.212232 \\
\hline $\mathrm{C}$ & 1.623740 & 1.237592 & 3.581477 \\
\hline $\mathrm{H}$ & -2.090758 & -0.945889 & -4.748358 \\
\hline $\mathrm{H}$ & -1.160544 & 1.422327 & -4.752980 \\
\hline $\mathrm{H}$ & -1.808902 & -2.099601 & -2.491611 \\
\hline $\mathrm{H}$ & -0.074797 & 3.026395 & -2.275351 \\
\hline $\mathrm{H}$ & 1.700533 & -1.493935 & -2.888564 \\
\hline $\mathrm{H}$ & 0.621513 & -3.636066 & -0.257828 \\
\hline $\mathrm{H}$ & 4.071281 & -2.266439 & -2.354762 \\
\hline $\mathrm{H}$ & 1.346368 & 4.131661 & -0.887330 \\
\hline $\mathrm{H}$ & -0.376826 & 4.466210 & -0.517897 \\
\hline $\mathrm{H}$ & -1.236254 & -5.100646 & 0.698008 \\
\hline $\mathrm{H}$ & 0.875026 & 4.673545 & 0.736779 \\
\hline $\mathrm{H}$ & 4.719855 & -1.817103 & 0.062997 \\
\hline $\mathrm{H}$ & -3.166297 & -3.736070 & 1.635243 \\
\hline $\mathrm{H}$ & 3.065440 & -0.210869 & 2.191826 \\
\hline $\mathrm{H}$ & -3.280775 & -0.603719 & 1.957444 \\
\hline $\mathrm{H}$ & -3.881633 & 1.601557 & 1.584906 \\
\hline $\mathrm{H}$ & 2.533094 & 1.747338 & 3.235935 \\
\hline $\mathrm{H}$ & -3.031431 & 2.909944 & 2.441022 \\
\hline $\mathrm{H}$ & 1.116724 & 1.859145 & 4.317292 \\
\hline $\mathrm{H}$ & 1.886665 & 0.282021 & 4.043841 \\
\hline $\mathrm{H}$ & -3.061246 & 1.276430 & 3.149151 \\
\hline $\mathrm{N}$ & -1.644728 & -0.517540 & -3.946588 \\
\hline $\mathrm{N}$ & -0.906026 & -0.251097 & -1.900622 \\
\hline $\mathrm{N}$ & 3.449133 & -1.842742 & -1.677537 \\
\hline $\mathrm{N}$ & 0.132629 & 1.728058 & -0.633300 \\
\hline $\mathrm{N}$ & 1.674303 & -0.866608 & -0.841960 \\
\hline $\mathrm{N}$ & -1.267752 & -4.088583 & 0.678261 \\
\hline $\mathrm{N}$ & -0.639044 & -2.017200 & 0.351720 \\
\hline $\mathrm{N}$ & 0.449982 & 2.675085 & 0.309000 \\
\hline $\mathrm{N}$ & 1.129593 & 0.160974 & 1.452715 \\
\hline $\mathrm{N}$ & -1.622712 & 0.260979 & 0.994100 \\
\hline $\mathrm{N}$ & 0.694480 & 0.973459 & 2.473859 \\
\hline $\mathrm{N}$ & -1.807400 & 1.547171 & 1.439678 \\
\hline $\mathrm{P}$ & -0.284746 & 2.316913 & 1.871565 \\
\hline S & -0.444081 & 3.817611 & 3.064896 \\
\hline $\mathrm{Fe}$ & -0.041773 & -0.177570 & -0.10746 \\
\hline
\end{tabular}


Table S3. Cartesian coordinates of complex $h s-[1]^{2+}(S=2 ;$ B3LYP*-D/TZVP).

\begin{tabular}{|c|c|c|c|}
\hline C & -1.275876 & 1.031213 & -4.052598 \\
\hline C & -1.221703 & -1.020961 & -3.216254 \\
\hline C & -0.793806 & 1.057030 & -2.767813 \\
\hline C & -0.438245 & 2.159805 & -1.904846 \\
\hline C & 2.497666 & -1.920847 & -1.646373 \\
\hline C & -0.801092 & -3.519468 & -0.104368 \\
\hline $\mathrm{C}$ & 0.181973 & 4.220336 & -0.150177 \\
\hline $\mathrm{C}$ & 4.045095 & -1.675161 & -0.077721 \\
\hline $\mathrm{C}$ & 2.908004 & -0.990411 & 0.270241 \\
\hline $\mathrm{C}$ & -2.565545 & -3.378184 & 1.230896 \\
\hline C & -2.008119 & -2.136121 & 1.047088 \\
\hline C & 2.599287 & -0.140264 & 1.396663 \\
\hline $\mathrm{C}$ & -2.335031 & -0.842306 & 1.599399 \\
\hline C & -2.918719 & 1.711028 & 2.515824 \\
\hline $\mathrm{C}$ & 1.932218 & 1.589421 & 3.453706 \\
\hline $\mathrm{H}$ & -1.929154 & -0.658421 & -5.17532 \\
\hline $\mathrm{H}$ & -1.463022 & 1.812553 & -4.772827 \\
\hline $\mathrm{H}$ & -1.350948 & -2.091379 & -3.155121 \\
\hline $\mathrm{H}$ & -0.448743 & 3.174141 & -2.29314 \\
\hline $\mathrm{H}$ & 2.032501 & -2.237723 & -2.56771 \\
\hline $\mathrm{H}$ & -0.042406 & -3.962717 & -0.73197 \\
\hline $\mathrm{H}$ & 4.402380 & -2.817190 & -1.84091 \\
\hline $\mathrm{H}$ & 0.920265 & 4.319105 & -0.95211 \\
\hline $\mathrm{H}$ & -0.795095 & 4.576278 & -0.50112 \\
\hline $\mathrm{H}$ & -1.919473 & -5.238489 & 0.42215 \\
\hline $\mathrm{H}$ & 0.499362 & 4.821402 & 0.69926 \\
\hline $\mathrm{H}$ & 4.998582 & -1.793774 & 0.41245 \\
\hline $\mathrm{H}$ & -3.413379 & -3.715190 & 1.80670 \\
\hline $\mathrm{H}$ & 3.342263 & 0.028682 & 2.16961 \\
\hline $\mathrm{H}$ & -3.212566 & -0.721875 & 2.22737 \\
\hline $\mathrm{H}$ & -3.773165 & 1.461629 & 1.87703 \\
\hline $\mathrm{H}$ & 2.823773 & 2.098234 & 3.06501 \\
\hline $\mathrm{H}$ & -2.930863 & 2.776101 & 2.736763 \\
\hline $\mathrm{H}$ & 1.425423 & 2.239729 & 4.163281 \\
\hline $\mathrm{H}$ & 2.225268 & 0.666127 & 3.96282 \\
\hline $\mathrm{H}$ & -2.982111 & 1.152132 & 3.457162 \\
\hline $\mathrm{N}$ & -1.542461 & -0.292681 & -4.313462 \\
\hline $\mathrm{N}$ & -0.760734 & -0.235994 & -2.262820 \\
\hline $\mathrm{N}$ & 3.762459 & -2.254526 & -1.293560 \\
\hline $\mathrm{N}$ & -0.171842 & 1.862212 & -0.675528 \\
\hline $\mathrm{N}$ & 1.951802 & -1.157902 & -0.72071 \\
\hline $\mathrm{N}$ & -1.784487 & -4.237227 & 0.495148 \\
\hline $\mathrm{N}$ & -0.909290 & -2.244297 & 0.20722 \\
\hline $\mathrm{N}$ & 0.126925 & 2.809733 & 0.25547 \\
\hline N & 1.428187 & 0.404884 & 1.385017 \\
\hline $\mathrm{N}$ & -1.511836 & 0.115919 & 1.321761 \\
\hline N & 1.006301 & 1.250088 & 2.364223 \\
\hline N & -1.691784 & 1.383910 & 1.780554 \\
\hline$P$ & -0.258059 & 2.374029 & 1.910401 \\
\hline S & -0.451881 & 3.884286 & 3.09700 \\
\hline $\mathrm{Fe}$ & 0.023070 & -0.325092 & -0.24292 \\
\hline
\end{tabular}


Table S4. Cartesian coordinates of complex Is-[2] $]^{2+}(S=0$; B3LYP*-D/TZVP).

\begin{tabular}{|c|c|c|c|}
\hline $\mathrm{N}$ & -1.213210 & 0.696208 & -3.726872 \\
\hline $\mathrm{C}$ & -1.464280 & -1.193222 & -2.605877 \\
\hline $\mathrm{C}$ & -0.769638 & 0.862940 & -2.455305 \\
\hline $\mathrm{C}$ & -0.251025 & 2.009885 & -1.774047 \\
\hline $\mathrm{C}$ & 2.091471 & -1.540446 & -1.860390 \\
\hline $\mathrm{C}$ & -0.476837 & -3.283224 & 0.049338 \\
\hline $\mathrm{C}$ & 0.482675 & 4.101023 & -0.087047 \\
\hline $\mathrm{N}$ & 3.696404 & -1.575842 & -0.339340 \\
\hline C & 2.609732 & -0.935021 & 0.162954 \\
\hline $\mathrm{N}$ & -2.509507 & -3.169838 & 0.911882 \\
\hline C & -1.942301 & -1.937975 & 0.926417 \\
\hline C & 2.390058 & -0.285025 & 1.418801 \\
\hline $\mathrm{C}$ & -2.431397 & -0.677190 & 1.395654 \\
\hline C & -2.956968 & 1.862416 & 2.412522 \\
\hline $\mathrm{C}$ & 1.776361 & 1.323133 & 3.596661 \\
\hline $\mathrm{C}$ & -1.254828 & 1.715821 & -4.776665 \\
\hline $\mathrm{H}$ & -1.682080 & -2.203015 & -2.295898 \\
\hline $\mathrm{H}$ & -0.234042 & 3.005276 & -2.200883 \\
\hline $\mathrm{H}$ & 1.490189 & -1.665151 & -2.747242 \\
\hline $\mathrm{H}$ & 0.448945 & -3.599074 & -0.405411 \\
\hline $\mathrm{H}$ & 1.226078 & 4.163820 & -0.887207 \\
\hline $\mathrm{H}$ & -0.488711 & 4.460004 & -0.451620 \\
\hline $\mathrm{H}$ & 0.807997 & 4.721103 & 0.746321 \\
\hline $\mathrm{C}$ & 4.995347 & -1.752730 & 0.315898 \\
\hline $\mathrm{C}$ & -3.839306 & -3.522450 & 1.415779 \\
\hline $\mathrm{H}$ & 3.166320 & -0.136585 & 2.158900 \\
\hline $\mathrm{H}$ & -3.333166 & -0.561948 & 1.983976 \\
\hline $\mathrm{H}$ & -3.831605 & 1.683920 & 1.779163 \\
\hline $\mathrm{H}$ & 2.666328 & 1.830633 & 3.201250 \\
\hline $\mathrm{H}$ & -2.930201 & 2.911809 & 2.699447 \\
\hline $\mathrm{H}$ & 1.300829 & 1.955353 & 4.344092 \\
\hline $\mathrm{H}$ & 2.063287 & 0.376324 & 4.062539 \\
\hline $\mathrm{H}$ & -3.013692 & 1.242852 & 3.316161 \\
\hline $\mathrm{C}$ & -1.659301 & -0.598540 & -3.832200 \\
\hline $\mathrm{N}$ & -0.922652 & -0.272489 & -1.757929 \\
\hline $\mathrm{C}$ & 3.382081 & -1.956524 & -1.620961 \\
\hline $\mathrm{N}$ & 0.097524 & 1.736109 & -0.546705 \\
\hline $\mathrm{N}$ & 1.626919 & -0.899014 & -0.749792 \\
\hline $\mathrm{C}$ & -1.586406 & -4.032441 & 0.370206 \\
\hline $\mathrm{N}$ & -0.706387 & -1.987718 & 0.407237 \\
\hline $\mathrm{N}$ & 0.419034 & 2.705261 & 0.366299 \\
\hline $\mathrm{N}$ & 1.181318 & 0.197967 & 1.517956 \\
\hline $\mathrm{N}$ & -1.608141 & 0.297307 & 1.120600 \\
\hline $\mathrm{N}$ & 0.799822 & 1.035499 & 2.536111 \\
\hline $\mathrm{N}$ & -1.759508 & 1.558978 & 1.622160 \\
\hline P & -0.229678 & 2.351113 & 1.969817 \\
\hline $\mathrm{S}$ & -0.353963 & 3.863947 & 3.151851 \\
\hline $\mathrm{Fe}$ & -0.044581 & -0.166566 & 0.004351 \\
\hline $\mathrm{H}$ & -1.790855 & -5.086619 & 0.265933 \\
\hline $\mathrm{H}$ & 4.092409 & -2.477803 & -2.243658 \\
\hline $\mathrm{H}$ & -2.076133 & -0.978328 & -4.751962 \\
\hline $\mathrm{H}$ & 5.497952 & -2.609669 & -0.133100 \\
\hline $\mathrm{H}$ & 4.845505 & -1.949832 & 1.379080 \\
\hline $\mathrm{H}$ & 5.612248 & -0.859504 & 0.182179 \\
\hline $\mathrm{H}$ & -1.583211 & 1.242896 & -5.701992 \\
\hline $\mathrm{H}$ & -0.259272 & 2.140403 & -4.924180 \\
\hline $\mathrm{H}$ & -1.961649 & 2.504428 & -4.506946 \\
\hline $\mathrm{H}$ & -4.141138 & -4.468289 & 0.966383 \\
\hline $\mathrm{H}$ & -4.553422 & -2.749645 & 1.123768 \\
\hline $\mathrm{H}$ & -3.821557 & -3.626783 & 2.503937 \\
\hline
\end{tabular}


Table S5. Cartesian coordinates of complex $h s-[2]^{2+}(S=2 ;$ B3LYP*-D/TZVP).

\begin{tabular}{|c|c|c|c|}
\hline $\mathrm{N}$ & -1.062800 & 0.684172 & -3.873476 \\
\hline $\mathrm{C}$ & -1.024641 & -1.347249 & -3.019298 \\
\hline $\mathrm{C}$ & -0.635380 & 0.740985 & -2.583852 \\
\hline $\mathrm{C}$ & -0.328011 & 1.906968 & -1.801365 \\
\hline $\mathrm{C}$ & 2.691277 & -1.996397 & -1.598908 \\
\hline $\mathrm{C}$ & -0.997416 & -3.641497 & -0.169203 \\
\hline $\mathrm{C}$ & 0.226430 & 4.062696 & -0.133672 \\
\hline $\mathrm{N}$ & 4.247026 & -1.398246 & -0.157237 \\
\hline C & 3.045959 & -0.904043 & 0.240705 \\
\hline $\mathrm{N}$ & -2.894114 & -3.218937 & 0.871160 \\
\hline C & -2.108108 & -2.112447 & 0.903931 \\
\hline C & 2.761175 & -0.068147 & 1.380137 \\
\hline $\mathrm{C}$ & -2.398785 & -0.840529 & 1.518466 \\
\hline $\mathrm{C}$ & -2.849480 & 1.676916 & 2.602737 \\
\hline $\mathrm{C}$ & 2.048043 & 1.547892 & 3.524052 \\
\hline $\mathrm{C}$ & -1.216538 & 1.807970 & -4.799530 \\
\hline $\mathrm{H}$ & -1.114023 & -2.408605 & -2.843570 \\
\hline $\mathrm{H}$ & -0.373958 & 2.900188 & -2.236402 \\
\hline $\mathrm{H}$ & 2.141079 & -2.412182 & -2.429123 \\
\hline $\mathrm{H}$ & -0.179110 & -4.098271 & -0.704240 \\
\hline $\mathrm{H}$ & 0.970790 & 4.144910 & -0.932085 \\
\hline $\mathrm{H}$ & -0.755455 & 4.376851 & -0.509940 \\
\hline $\mathrm{H}$ & 0.519387 & 4.708303 & 0.691575 \\
\hline $\mathrm{C}$ & 5.558270 & -1.156236 & 0.451472 \\
\hline $\mathrm{C}$ & -4.232268 & -3.372477 & 1.449886 \\
\hline $\mathrm{H}$ & 3.535716 & 0.182127 & 2.097019 \\
\hline $\mathrm{H}$ & -3.327993 & -0.687147 & 2.057063 \\
\hline $\mathrm{H}$ & -3.676507 & 1.566118 & 1.892205 \\
\hline $\mathrm{H}$ & 2.884560 & 2.135925 & 3.124114 \\
\hline $\mathrm{H}$ & -2.784473 & 2.713084 & 2.927232 \\
\hline $\mathrm{H}$ & 1.521388 & 2.134750 & 4.273430 \\
\hline $\mathrm{H}$ & 2.424506 & 0.631249 & 3.987566 \\
\hline $\mathrm{H}$ & -3.023535 & 1.041052 & 3.478482 \\
\hline $\mathrm{C}$ & -1.307632 & -0.635702 & -4.161594 \\
\hline $\mathrm{N}$ & -0.608745 & -0.482861 & -2.047108 \\
\hline $\mathrm{C}$ & 4.034485 & -2.091685 & -1.323955 \\
\hline $\mathrm{N}$ & -0.046327 & 1.671793 & -0.557967 \\
\hline $\mathrm{N}$ & 2.086075 & -1.259568 & -0.621853 \\
\hline $\mathrm{C}$ & -2.196540 & -4.196542 & 0.200989 \\
\hline $\mathrm{N}$ & -0.951583 & -2.347204 & 0.269081 \\
\hline $\mathrm{N}$ & 0.204526 & 2.669857 & 0.330963 \\
\hline $\mathrm{N}$ & 1.542549 & 0.365334 & 1.455882 \\
\hline $\mathrm{N}$ & -1.474971 & 0.063164 & 1.396872 \\
\hline $\mathrm{N}$ & 1.106156 & 1.169114 & 2.460625 \\
\hline $\mathrm{N}$ & -1.600432 & 1.297710 & 1.932761 \\
\hline P & -0.166196 & 2.290900 & 2.002281 \\
\hline $\mathrm{S}$ & -0.330772 & 3.830925 & 3.151258 \\
\hline $\mathrm{Fe}$ & 0.148034 & -0.567627 & -0.054087 \\
\hline $\mathrm{H}$ & 4.841078 & -2.583517 & -1.844960 \\
\hline $\mathrm{H}$ & -1.667011 & -0.946504 & -5.130229 \\
\hline $\mathrm{H}$ & -2.609388 & -5.181399 & 0.049365 \\
\hline $\mathrm{H}$ & -4.715898 & -4.226018 & 0.975375 \\
\hline $\mathrm{H}$ & -4.823604 & -2.477077 & 1.246985 \\
\hline $\mathrm{H}$ & -4.167459 & -3.546267 & 2.527344 \\
\hline $\mathrm{H}$ & -1.892090 & 1.507245 & -5.600532 \\
\hline $\mathrm{H}$ & -0.250115 & 2.088476 & -5.225917 \\
\hline $\mathrm{H}$ & -1.653890 & 2.658445 & -4.273302 \\
\hline $\mathrm{H}$ & 6.142671 & -2.076939 & 0.412719 \\
\hline $\mathrm{H}$ & 5.429812 & -0.860563 & 1.492950 \\
\hline $\mathrm{H}$ & 6.081785 & -0.365680 & -0.091879 \\
\hline
\end{tabular}


Table S6. Cartesian coordinates of complex $/ s-[3]^{2+}(S=0$; B3LYP*-D/TZVP).

\begin{tabular}{|c|c|c|c|}
\hline C & 0.900148 & -1.590988 & -3.620593 \\
\hline $\mathrm{C}$ & 1.656603 & -2.705794 & -3.297231 \\
\hline $\mathrm{C}$ & -1.261547 & 3.063261 & -3.207844 \\
\hline C & -0.206275 & 0.522018 & -2.870538 \\
\hline C & 0.503368 & -0.707864 & -2.617460 \\
\hline C & 2.002073 & -2.914052 & -1.959234 \\
\hline $\mathrm{C}$ & 1.573216 & -1.985975 & -0.998219 \\
\hline $\mathrm{C}$ & 2.520015 & 3.816556 & -0.340038 \\
\hline $\mathrm{C}$ & -1.821884 & -2.041740 & -0.269376 \\
\hline $\mathrm{C}$ & -2.907350 & -2.821914 & 0.146727 \\
\hline C & -3.827180 & -2.272846 & 1.047857 \\
\hline C & 2.335628 & 1.370811 & 0.973901 \\
\hline $\mathrm{C}$ & -2.546319 & -0.238752 & 1.003223 \\
\hline C & -2.326725 & 1.154281 & 1.310968 \\
\hline $\mathrm{C}$ & -3.641900 & -0.969450 & 1.468242 \\
\hline $\mathrm{C}$ & -1.909142 & 3.899789 & 1.515625 \\
\hline $\mathrm{C}$ & 2.068577 & 0.179084 & 1.744650 \\
\hline $\mathrm{C}$ & 0.477436 & -1.430952 & 2.264722 \\
\hline C & 2.940257 & -0.329521 & 2.710772 \\
\hline $\mathrm{C}$ & 1.297818 & -1.977137 & 3.259460 \\
\hline $\mathrm{C}$ & 2.562958 & -1.419168 & 3.47144 \\
\hline $\mathrm{H}$ & 0.614239 & -1.395233 & -4.64704 \\
\hline $\mathrm{H}$ & 1.976725 & -3.402323 & -4.06497 \\
\hline $\mathrm{H}$ & -0.370806 & 3.114278 & -3.84468 \\
\hline $\mathrm{H}$ & -0.402646 & 0.857988 & -3.88083 \\
\hline $\mathrm{H}$ & -2.014317 & 2.422461 & -3.67370 \\
\hline $\mathrm{H}$ & -1.670636 & 4.062374 & -3.07913 \\
\hline $\mathrm{H}$ & 3.012950 & -4.534947 & -2.22004 \\
\hline $\mathrm{H}$ & -2.444647 & -4.371293 & -0.90967 \\
\hline $\mathrm{H}$ & -1.083026 & -2.447354 & -0.95027 \\
\hline $\mathrm{H}$ & 2.342842 & 4.709393 & -0.93432 \\
\hline $\mathrm{H}$ & 3.426018 & 3.319813 & -0.69568 \\
\hline $\mathrm{H}$ & 1.835885 & -2.134277 & 0.04048 \\
\hline $\mathrm{H}$ & 2.640457 & 4.100046 & 0.71212 \\
\hline $\mathrm{H}$ & -2.919612 & 3.861646 & 1.09165 \\
\hline $\mathrm{H}$ & -4.666366 & -2.868291 & 1.38669 \\
\hline $\mathrm{H}$ & 3.201881 & 1.987090 & 1.18087 \\
\hline $\mathrm{H}$ & -1.527203 & 4.915666 & 1.44678 \\
\hline $\mathrm{H}$ & -3.066864 & 1.733990 & 1.84803 \\
\hline $\mathrm{H}$ & -4.342077 & -0.505378 & 2.15235 \\
\hline $\mathrm{H}$ & -0.498657 & -1.857222 & 2.06669 \\
\hline $\mathrm{H}$ & -1.934673 & 3.596149 & 2.56510 \\
\hline $\mathrm{H}$ & 3.905479 & 0.138590 & 2.86044 \\
\hline $\mathrm{H}$ & 0.033284 & -3.317968 & 3.83909 \\
\hline $\mathrm{H}$ & 3.212295 & -1.841988 & 4.228475 \\
\hline $\mathrm{N}$ & -0.951431 & 2.510459 & -1.884840 \\
\hline $\mathrm{N}$ & -0.484583 & 1.225552 & -1.81858 \\
\hline $\mathrm{N}$ & 0.845171 & -0.915024 & -1.31406 \\
\hline $\mathrm{N}$ & 1.391338 & 2.899842 & -0.53167 \\
\hline N & 1.420528 & 1.692921 & 0.114422 \\
\hline $\mathrm{N}$ & -1.640543 & -0.784350 & 0.14757 \\
\hline $\mathrm{N}$ & -0.989329 & 3.008074 & 0.80308 \\
\hline $\mathrm{N}$ & -1.249728 & 1.666379 & 0.80413 \\
\hline $\mathrm{N}$ & 0.850049 & -0.383509 & 1.52380 \\
\hline O & 2.715635 & -3.957116 & -1.50366 \\
\hline O & -3.106533 & -4.084438 & -0.26654 \\
\hline O & 0.934001 & -3.019607 & 4.02117 \\
\hline $\mathrm{P}$ & -0.228763 & 3.538966 & -0.67292 \\
\hline S & -0.340009 & 5.415098 & -1.01821 \\
\hline $\mathrm{Fe}$ & -0.045425 & 0.406782 & -0.09343 \\
\hline
\end{tabular}


Table S7. Cartesian coordinates of complex $h s-[3]^{2+}\left(S=2 ; B 3 L Y{ }^{*}-D / T Z V P\right)$.

\begin{tabular}{|c|c|c|c|}
\hline C & 0.768839 & -1.629219 & -3.734115 \\
\hline $\mathrm{C}$ & 1.289717 & -2.889903 & -3.486999 \\
\hline C & -0.802652 & 3.203937 & -3.295798 \\
\hline C & 0.060509 & 0.603037 & -2.891469 \\
\hline C & 0.530161 & -0.756518 & -2.671424 \\
\hline C & 1.577486 & -3.248608 & -2.168445 \\
\hline $\mathrm{C}$ & 1.291248 & -2.328053 & -1.150642 \\
\hline $\mathrm{C}$ & 2.451324 & 4.102480 & 0.138165 \\
\hline C & -1.901682 & -2.206849 & 0.205815 \\
\hline C & -3.076758 & -2.908114 & 0.500108 \\
\hline C & -4.160494 & -2.199222 & 1.026804 \\
\hline C & 2.369935 & 1.620469 & 1.386480 \\
\hline $\mathrm{C}$ & -2.833776 & -0.197776 & 0.881276 \\
\hline C & -2.694510 & 1.247755 & 0.980743 \\
\hline $\mathrm{C}$ & -4.031880 & -0.835520 & 1.214149 \\
\hline $\mathrm{C}$ & -2.448566 & 4.005521 & 1.050891 \\
\hline $\mathrm{C}$ & 2.314291 & 0.291225 & 1.979240 \\
\hline $\mathrm{C}$ & 1.098650 & -1.668180 & 2.230930 \\
\hline C & 3.307258 & -0.182115 & 2.841401 \\
\hline $\mathrm{C}$ & 2.044395 & -2.199284 & 3.116963 \\
\hline $\mathrm{C}$ & 3.178553 & -1.435777 & 3.411054 \\
\hline $\mathrm{H}$ & 0.551573 & -1.314332 & -4.747772 \\
\hline $\mathrm{H}$ & 1.482361 & -3.580117 & -4.301821 \\
\hline $\mathrm{H}$ & 0.052981 & 3.176127 & -3.979383 \\
\hline $\mathrm{H}$ & -0.151499 & 0.920384 & -3.90694 \\
\hline $\mathrm{H}$ & -1.613474 & 2.591087 & -3.70243 \\
\hline $\mathrm{H}$ & -1.142273 & 4.230650 & -3.18880 \\
\hline $\mathrm{H}$ & 2.303570 & -4.997451 & -2.537212 \\
\hline $\mathrm{H}$ & -2.439996 & -4.627118 & -0.10752 \\
\hline $\mathrm{H}$ & -1.044701 & -2.724879 & -0.21311 \\
\hline $\mathrm{H}$ & 2.242255 & 4. 992162 & -0.45026 \\
\hline $\mathrm{H}$ & 3.320497 & 3.582826 & -0.27759 \\
\hline $\mathrm{H}$ & 1.501323 & -2.591027 & -0.12054 \\
\hline $\mathrm{H}$ & 2.659438 & 4.397445 & 1.17154 \\
\hline $\mathrm{H}$ & -3.357557 & 3.915958 & 0.44523 \\
\hline $\mathrm{H}$ & -5.078005 & -2.723589 & 1.266040 \\
\hline $\mathrm{H}$ & 3.225873 & 2.254415 & 1.593717 \\
\hline $\mathrm{H}$ & -2.093703 & 5.032040 & 1.017937 \\
\hline $\mathrm{H}$ & -3.532542 & 1.825303 & 1.35577 \\
\hline $\mathrm{H}$ & -4.857768 & -0.253834 & 1.605688 \\
\hline $\mathrm{H}$ & 0.205802 & -2.228345 & 1.96739 \\
\hline $\mathrm{H}$ & -2.666727 & 3.731634 & $2.08660^{\circ}$ \\
\hline $\mathrm{H}$ & 4.167456 & 0.437093 & 3.065563 \\
\hline $\mathrm{H}$ & 1.090181 & -3.838217 & 3.48776 \\
\hline $\mathrm{H}$ & 3.927665 & -1.835975 & 4.083860 \\
\hline $\mathrm{N}$ & -0.422444 & 2.679236 & -1.982007 \\
\hline N & -0.043100 & 1.386935 & -1.86863 \\
\hline N & 0.771039 & -1.128896 & -1.392962 \\
\hline N & 1.293149 & 3.205456 & 0.08269 \\
\hline N & 1.365874 & 1.989979 & 0.66508 \\
\hline N & -1.783741 & -0.890869 & 0.38961 \\
\hline N & -1.394763 & 3.118315 & 0.54482 \\
\hline N & -1.595934 & 1.778273 & 0.56365 \\
\hline N & 1.239116 & -0.464497 & 1.67347 \\
\hline O & 2.096261 & -4.427694 & -1.78359 \\
\hline O & -3.217722 & -4.229476 & 0.30519 \\
\hline O & 1.931932 & -3.408718 & 3.68904 \\
\hline $\mathrm{P}$ & -0.218954 & 3.695445 & -0.59076 \\
\hline S & -0.342624 & 5.569282 & -0.97474 \\
\hline $\mathrm{Fe}$ & 0.007813 & 0.328579 & 0.06603 \\
\hline
\end{tabular}


Table S8. Cartesian coordinates of complex $I s-[4]^{2+}(S=0$; B3LYP*-D/TZVP).

\begin{tabular}{|c|c|c|c|}
\hline $\mathrm{Fe}$ & -1.902535 & -8.251354 & -12.018685 \\
\hline $\mathrm{N}$ & -3.877374 & -6.255741 & -14.025588 \\
\hline $\mathrm{C}$ & -3.173260 & -5.078323 & -13.602250 \\
\hline $\mathrm{H}$ & -3.040799 & -4.371521 & -14.434853 \\
\hline $\mathrm{H}$ & -3.772759 & -4.566987 & -12.841970 \\
\hline $\mathrm{N}$ & -1.809112 & -6.252842 & -11.885261 \\
\hline C & -1.765974 & -5.342221 & -13.023474 \\
\hline $\mathrm{H}$ & -1.316553 & -4.383147 & -12.737205 \\
\hline $\mathrm{H}$ & -1.140654 & -5.803512 & -13.791402 \\
\hline $\mathrm{N}$ & -1.861365 & -8.060821 & -10.029379 \\
\hline $\mathrm{C}$ & -1.724300 & -5.772023 & -10.692160 \\
\hline $\mathrm{H}$ & -1.604726 & -4.705599 & -10.498930 \\
\hline $\mathrm{N}$ & -1.910001 & -7.982455 & -7.840137 \\
\hline $\mathrm{C}$ & -1.787781 & -6.733643 & -9.624103 \\
\hline $\mathrm{C}$ & -1.937427 & -8.789676 & -8.937310 \\
\hline $\mathrm{H}$ & -2.016124 & -9.864889 & -8.887470 \\
\hline C & -1.815110 & -6.677286 & -8.250981 \\
\hline $\mathrm{H}$ & -1.781598 & -5.850418 & -7.558391 \\
\hline C & -3.451977 & -6.961216 & -15.201428 \\
\hline $\mathrm{H}$ & -4.280508 & -7.084354 & -15.914733 \\
\hline $\mathrm{H}$ & -2.682346 & -6.367772 & -15.705709 \\
\hline $\mathrm{N}$ & -1.756467 & -8.342908 & -14.016742 \\
\hline $\mathrm{C}$ & -2.888320 & -8.374268 & -14.935864 \\
\hline $\mathrm{H}$ & -2.598915 & -8.824644 & -15.893540 \\
\hline $\mathrm{H}$ & -3.665396 & -8.993217 & -14.481449 \\
\hline $\mathrm{N}$ & 0.087800 & -8.308741 & -12.195627 \\
\hline $\mathrm{C}$ & -0.560721 & -8.437055 & -14.489202 \\
\hline $\mathrm{H}$ & -0.360889 & -8.556327 & -15.554451 \\
\hline $\mathrm{N}$ & 2.277922 & -8.279683 & -12.259684 \\
\hline $\mathrm{C}$ & 0.501156 & -8.384075 & -13.520185 \\
\hline $\mathrm{C}$ & 1.175780 & -8.243627 & -11.459582 \\
\hline $\mathrm{H}$ & 1.219288 & -8.167049 & -10.383931 \\
\hline $\mathrm{C}$ & 1.874796 & -8.368863 & -13.567590 \\
\hline $\mathrm{H}$ & 2.572465 & -8.407598 & -14.389952 \\
\hline $\mathrm{C}$ & -5.061678 & -6.672241 & $-13 \cdot 329038$ \\
\hline $\mathrm{H}$ & -5.768407 & -5.837673 & -13.208906 \\
\hline $\mathrm{H}$ & -5.568776 & -7.436016 & -13.927647 \\
\hline $\mathrm{N}$ & -3.901914 & -8.381166 & -11.941689 \\
\hline $\mathrm{C}$ & -4.811223 & -7.241294 & -11.915308 \\
\hline $\mathrm{H}$ & -5.774732 & -7.522893 & -11.472474 \\
\hline $\mathrm{H}$ & -4.354495 & -6.469414 & -11.291533 \\
\hline $\mathrm{N}$ & -2.096193 & -10.239847 & -11.965109 \\
\hline $\mathrm{C}$ & -4.385117 & -9.572908 & -11.851564 \\
\hline $\mathrm{H}$ & -5.452829 & -9.763741 & -11.739773 \\
\hline $\mathrm{N}$ & -2.177279 & -12.429274 & -11.996460 \\
\hline $\mathrm{C}$ & -3.424503 & -10.642730 & -11.898702 \\
\hline $\mathrm{C}$ & -1.368296 & -11.333485 & -12.026454 \\
\hline $\mathrm{H}$ & -0.292538 & -11.385481 & -12.095964 \\
\hline $\mathrm{C}$ & -3.482558 & -12.015961 & -11.915175 \\
\hline $\mathrm{H}$ & -4.310624 & -12.707143 & -11.882253 \\
\hline $\mathrm{H}$ & -1.956828 & -8.296306 & -6.878305 \\
\hline $\mathrm{H}$ & -1.864504 & -13.391879 & -12.033395 \\
\hline $\mathrm{H}$ & 3.237938 & -8.241321 & -11.939214 \\
\hline
\end{tabular}


Table S9. Cartesian coordinates of complex $h s-[4]^{2+}\left(S=2 ; B 3 L Y{ }^{*}-D / T Z V P\right)$.

\begin{tabular}{|c|c|c|c|}
\hline $\mathrm{Fe}$ & -2.045647 & -8.109514 & -12.201000 \\
\hline $\mathrm{N}$ & -3.689443 & -6.440191 & -13.861291 \\
\hline $\mathrm{C}$ & -3.430764 & -5.083972 & -13.375747 \\
\hline $\mathrm{H}$ & -3.575100 & -4.328418 & -14.162091 \\
\hline $\mathrm{H}$ & -4.136518 & -4.856308 & -12.571803 \\
\hline $\mathrm{N}$ & -1.856579 & -5.985431 & -11.770170 \\
\hline $\mathrm{C}$ & -2.008315 & -4.979728 & -12.812470 \\
\hline $\mathrm{H}$ & -1.821204 & -3.965952 & -12.435352 \\
\hline $\mathrm{H}$ & -1.282999 & -5.195233 & -13.604584 \\
\hline $\mathrm{N}$ & -1.839251 & -7.987464 & -9.962840 \\
\hline C & -1.687084 & -5.633936 & -10.549851 \\
\hline $\mathrm{H}$ & -1.586798 & -4.587357 & -10.249735 \\
\hline $\mathrm{N}$ & -1.644376 & -7.938366 & -7.773123 \\
\hline C & -1.664506 & -6.674120 & -9.550048 \\
\hline $\mathrm{C}$ & -1.822124 & -8.721660 & -8.874796 \\
\hline $\mathrm{H}$ & -1.940480 & -9.793948 & -8.824740 \\
\hline $\mathrm{C}$ & -1.544094 & -6.634275 & -8.179909 \\
\hline $\mathrm{H}$ & -1.402550 & -5.817295 & -7.488853 \\
\hline $\mathrm{C}$ & -3.212840 & -6.689045 & -15.222240 \\
\hline $\mathrm{H}$ & -4.003494 & -6.540044 & -15.972425 \\
\hline $\mathrm{H}$ & -2.411001 & -5.980000 & -15.448465 \\
\hline $\mathrm{N}$ & -1.606162 & -8.271993 & -14.339468 \\
\hline $\mathrm{C}$ & -2.647996 & -8.109909 & -15.342969 \\
\hline $\mathrm{H}$ & -2.271496 & -8.285562 & -16.359138 \\
\hline $\mathrm{H}$ & -3.437927 & -8.838784 & -15.132540 \\
\hline $\mathrm{N}$ & 0.210216 & -8.362927 & -12.324141 \\
\hline C & -0.387872 & -8.461613 & -14.682451 \\
\hline $\mathrm{H}$ & -0.084724 & -8.565377 & -15.728543 \\
\hline $\mathrm{N}$ & 2.401082 & -8.552562 & -12.394532 \\
\hline C & 0.614194 & -8.510218 & -13.642443 \\
\hline $\mathrm{C}$ & 1.303634 & -8.391182 & -11.600331 \\
\hline $\mathrm{H}$ & 1.362151 & -8.297426 & -10.525747 \\
\hline $\mathrm{C}$ & 1.983567 & -8.628395 & -13.696933 \\
\hline $\mathrm{H}$ & 2.668523 & -8.750521 & -14.522343 \\
\hline $\mathrm{C}$ & -5.046971 & -6.920445 & -13.601942 \\
\hline $\mathrm{H}$ & -5.802408 & -6.140425 & -13.779804 \\
\hline $\mathrm{H}$ & -5.263000 & -7.746816 & -14.285492 \\
\hline $\mathrm{N}$ & -4.188482 & -8.492027 & -11.968883 \\
\hline C & -5.171307 & -7.437431 & -12.163308 \\
\hline $\mathrm{H}$ & -6.194468 & -7.788384 & -11.975000 \\
\hline $\mathrm{H}$ & -4.945777 & -6.627581 & -11.461512 \\
\hline $\mathrm{N}$ & -2.225838 & -10.334799 & -11.903103 \\
\hline C & -4.561465 & -9.689152 & -11.713408 \\
\hline $\mathrm{H}$ & -5.610410 & -9.961356 & -11.566175 \\
\hline $\mathrm{N}$ & -2.308364 & -12.510939 & -11.612143 \\
\hline C & -3.538798 & -10.710693 & -11.661350 \\
\hline $\mathrm{C}$ & -1.512142 & -11.436392 & -11.867794 \\
\hline $\mathrm{H}$ & -0.446126 & -11.513490 & -12.023417 \\
\hline $\mathrm{C}$ & -3.600865 & -12.071486 & -11.477915 \\
\hline $\mathrm{H}$ & -4.424760 & -12.738767 & -11.275470 \\
\hline $\mathrm{H}$ & -1.609698 & -8.263405 & -6.814299 \\
\hline $\mathrm{H}$ & -2.001424 & -13.474266 & -11.547512 \\
\hline $\mathrm{H}$ & 3.361942 & -8.598051 & -12.076545 \\
\hline
\end{tabular}

